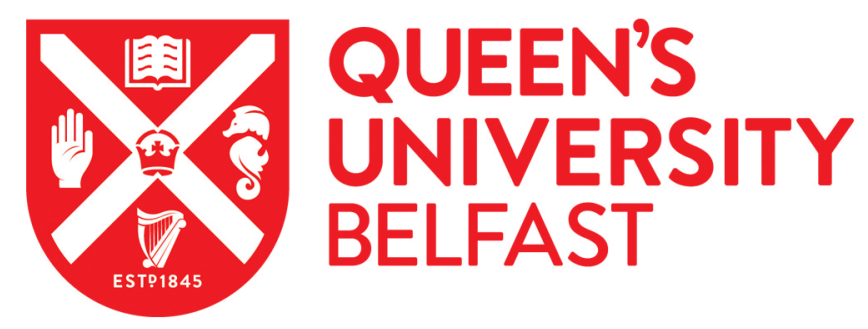

\title{
Lens-loaded Cavity Antenna with Detector Diode as a Direction-of- Arrival Estimator
}

Abbasi, M. A. B., Fusco, V., Yurduseven, O., Ansari, R. I., \& Cotton, S. (2021). Lens-loaded Cavity Antenna with Detector Diode as a Direction-of-Arrival Estimator. IEEE Antennas and Wireless Propagation Letters.

https://doi.org/10.1109/LAWP.2021.3094363

Published in:

IEEE Antennas and Wireless Propagation Letters

Document Version:

Peer reviewed version

Queen's University Belfast - Research Portal:

Link to publication record in Queen's University Belfast Research Portal

Publisher rights

Copyright 2021, IEEE.

This work is made available online in accordance with the publisher's policies. Please refer to any applicable terms of use of the publisher.

\section{General rights}

Copyright for the publications made accessible via the Queen's University Belfast Research Portal is retained by the author(s) and / or other copyright owners and it is a condition of accessing these publications that users recognise and abide by the legal requirements associated with these rights.

Take down policy

The Research Portal is Queen's institutional repository that provides access to Queen's research output. Every effort has been made to ensure that content in the Research Portal does not infringe any person's rights, or applicable UK laws. If you discover content in the Research Portal that you believe breaches copyright or violates any law, please contact openaccess@qub.ac.uk. 


\title{
Lens-loaded Cavity Antenna with Detector Diode as a Direction-of-Arrival Estimator
}

\author{
M. Ali Babar Abbasi Member, IEEE, Vincent Fusco, Fellow, IEEE, Okan Yurduseven, Senior Member, IEEE, \\ Rafay I. Ansari Member, IEEE, and Simon L. Cotton, Senior Member, IEEE
}

\begin{abstract}
This letter proposes a novel direction-of-arrival (DoA) estimation approach which uses a lens-loaded, coded aperture antenna connected to a detector diode for millimeterwave (mmWave) frequencies. The presented approach exploits three distinct, but closely related properties of mmWave communication front-end hardware: First, the quasi-random radiation modes created by a lens-loaded cavity antenna; Second, the frequency-diverse impulse response of over-sized metallic cavities; Third, the sensitivity of detector diodes at mmWave frequencies. Critically, we use a combination of the synthesis approach and experimental results to demonstrate that DoA estimation is possible with less complex hardware as compared to conventional methods, where an array of mmWave antennas is typically employed for DoA estimation. Bandwidth-to-accuracy trade-off is thoroughly studied, which is an important commercial matrix. It is demonstrated that the lens-loaded cavity antenna with a detector diode circuit can be an apt choice for DoA estimation. Finally, it is shown that the proposed approach provides fast and accurate DoA estimation, thus inherently suitable for DoA estimation in future mmWave systems.
\end{abstract}

Index Terms-B5G, channel estimation, detector diode, direction-of-arrival estimation, frequency-diverse antenna, lens antenna, millimetre-wave.

\section{INTRODUCTION}

A CCURATE direction-of-arrival (DoA) information is a key requisite of mmWave channel sounding. Typically, an array of antennas and associated radio frequency (RF) hardware is employed for DoA estimation, in conjunction with techniques such as the ESPRIT [1], Capon [2], Bartlett [3] and MUSIC [4], [5] algorithms. The development of antenna arrays can be both complex and costly at mmWave frequencies. Notwithstanding the number of antennas required to provide sufficient angular discrimination, high path loss at mmWave frequencies requires a greater number of antennas in an array formation, thereby increasing the complexity of radio hardware. Recently, a number of mmWave antenna hardware simplification approaches have been investigated, which focus on classical beam synthesis [3], [6]-[9]. Highly directive,

Manuscript received MM DD, 2021. This work was partially funded by the Engineering and Physical Sciences Research Council (EPSRC) under grant EP/P000673/1 and Leverhulme Trust under Research Leadership Award RL2019-019.

M.A.B. Abbasi, V. Fusco, O. Yurduseven and S. L. Cotton are with the Institute of Electronics, Communications and Information Technology (ECIT), Queen's University Belfast, Belfast BT3 9DT, United Kingdom. Email: m.Abbasi@qub.ac.uk; v.fusco@ecit.qub.ac.uk; okan.yurduseven@qub.ac.uk; simon.cotton@qub.ac.uk

R. I. Ansari is with the Department of Computer and Information Sciences, Northumbria University, Newcastle upon Tyne NE1 8ST, United Kingdom. Email: rafay.ansari@northumbria.ac.uk

Corresponding author: M. A. B. Abbasi.

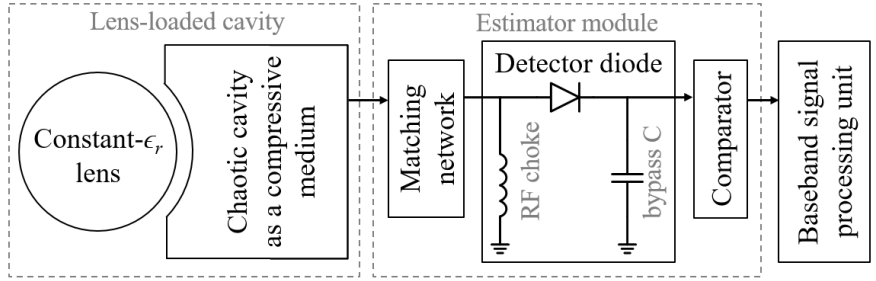

Fig. 1: System block diagram showing lens-loaded cavity antenna and estimator module.

frequency-diverse antenna apertures have also been shown to be a promising alternative to a fully connected antenna array. Frequency diverse antenna apertures use microwave computational imaging concepts (e.g. [10]-[14]), where fieldof-view (FoV) information is captured. Recently, it has been shown that channel information within a FoV (in terms of far-field radiation) can also be constructed from quasi-random measurement modes using computational techniques [15]. In this context, in [15], a preliminary theoretical investigation of DoA estimation using a mode-mixing cavity was presented. However, this was limited by the use of a hypothetical frequency-diverse antenna with high-Q factor. In [16], a numerical and experimental validation of DoA estimation using a lens-loaded cavity aperture was presented. These works use computational methods for the DoA estimation.

This work proposes a novel DoA estimation approach that requires a lens-loaded frequency-diverse antenna connected to a detector diode circuit as a mmWave receiver. One of the main drawbacks of the aforementioned studied techniques is their reliance on a high bandwidth. In this contribution, we show that DoA estimation is possible using significantly less bandwidth compared to computational techniques proposed in [15] and [16]. This is made possible using the proposed estimation technique which exploits the classical concept of power detection to translate the DoA information at the output of a lens-loaded cavity antenna into a detectable voltage.

\section{Proposed DoA Estimation Technique}

The proposed system block diagram is given in Fig. 1, where a lens-loaded cavity is connected to a base-band signal processing unit via an estimator module. The lens loaded cavity is composed of an over-sized metallic hollow cavity with a curved opening comprising of an array of sub-wavelength holes. The cavity opening is covered with a constant- $\epsilon_{r}$ lens [17] that provides a high antenna gain. Typically, this required lens operation can be achieved when the dielectric constant $\left(\epsilon_{r}\right)$ value of the lens material is more than 2.0 and less 


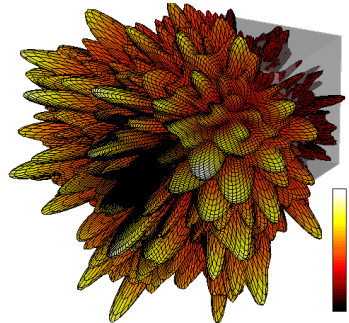

(a)

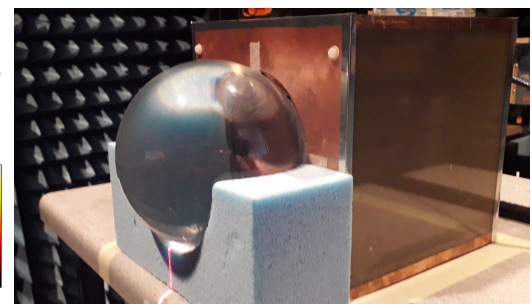

(b)
Fig. 2: (a) Lens-loaded cavity antenna far-field gain patterns (color map is scaled from -27.8 to $12.2 \mathrm{dBi}$ ). (b) Photograph of fabricated lens-loaded cavity.

than 3.5. The lens structure used in this study has $\epsilon_{r}=2.53$ which makes the focal point $3.5 \mathrm{~mm}$. The lens is developed by machining out a sphere from Rexolite material having a loss $\operatorname{tangent} \tan \delta=0.00066$. Choice of lens material with this loss tangent means the wave propagating through the lens structure will suffer significantly less attenuation compared to the gain achieved due to lens operation. The mode mixing cavity has dimensions of approximately $18 \times 18 \times 18 \mathrm{~cm}^{3}$, while the Rexolite lens has a diameter of $13.3 \mathrm{~cm}$. For a standard base station application, $18 \times 18 \mathrm{~cm}^{2}$ aperture size is practical. An arbitrarily oriented metallic scatterer of size around $3 \times 5 \mathrm{~cm}^{2}$ is placed inside the chaotic cavity to introduce and enhance the mode-mixing capabilities by introducing quasi-random disorderly reflections. The metallic scatterer built inside that helps mode-mixing and also breaks the structural symmetry. The randomly oriented scatterer produces enough disorder in the chaotic cavity that for a specific fabricated lens-loaded cavity, the quasi-random radiation modes are very subjective and device specific. A detailed explanation of the construction of the lens-loaded cavity and rationale behind the use of the lens can be found in [16]. The estimator module in Fig. 1 comprises of an impedance matching network, a detector diode circuit, and a comparator system. The detector diode circuit is a two-port microwave device that rectifies a received radio signal. This is similar to the detectors widely used in amplitude modulation based radio receivers.

The primary objective of the detector diode circuit in Fig. 1 is to provide a voltage amplitude that is proportional to the received power at the output of the lens-loaded cavity antenna. Typically, an RF choke is connected in shunt and provides the required DC return necessary for the detector diode to provide the $\mathrm{DC}$ voltage to the comparator. The RF choke acts as an open circuit for the signal coming into the detector diode circuit. The shunt capacitance after the detector diode provides ground to the diode, which acts as an open circuit at detector's baseband bandwidth. Detector diode circuits are often packaged into power meters operating as a scalar network analyzers (e.g. Keysight N1922A and N8480). The detector diode block input impedance is generally not a good match to the conjugate of the lens-loaded cavity output, therefore, a matching network operating as an impedance transformer is required between the both. The output voltage (V) is directly proportional to the square root of the input signal power (W). The sensitivity of the detector diode is given in the form of an open-circuit voltage in $\mathrm{mV}$ per unit $\mathrm{mW}$.
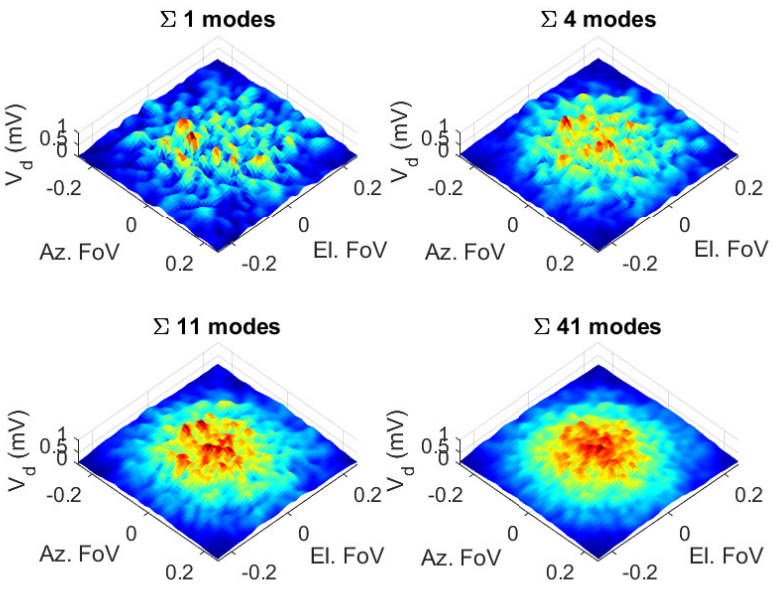

Fig. 3: Normalized voltage map at the output of detector diode circuit when normalized sum of multiple modes are used (color map scale $0-1 \mathrm{mV}$ where red and blue are maximum and minimum respectively).

Hence, the estimator sensitivity $S_{\text {est }}$ is given by

$$
S_{e s t}\left(P_{a v, c}\right)=\frac{V_{d}}{P_{a v, d}(\theta, \phi)}(\mathrm{mV} / \mathrm{mW}),
$$

where $P_{a v, c}$ is the received signal power available at the lensloaded cavity, $\theta$ and $\phi$ are the angular variation along elevation and azimuth directions within the $\mathrm{FoV}, V_{d}$ is the estimator output voltage, and $P_{a v, d}$ is the power available at the input of the detector diode circuit.

The absolute $V_{d}$ value depends on the load resistance, however, for the sake of simplicity and generality we used $V_{d}$ values normalized to $1 \mathrm{mV}$ in this study. Generally the detector diodes operate at a 0-bias, hence, a very low input power level at the output of the lens-loaded cavity can lead to estimator sensitivity deviation. One possible way to mitigate this deviation is to bias the detector diode circuit to match the comparator's voltage levels. For a given voltage level of the detector diode circuit, the comparator creates a binary output signal and provides it to the digital signal processing block. In the sequel, we present the hardware construction of the system architecture presented in Fig. 1 that can be used for DoA estimation.

\section{MeAsurements, Results AND Discussion}

A proof-of-concept mmWave lens-loaded cavity hardware is used to investigate and demonstrate the proposed approach. When a mmWave signal excites the input of the lens loaded cavity, spatially diverse radiation patterns are observed (Fig. 2(a) shows an example of this at a frequency of $28 \mathrm{GHz}$ ). Conversely, impinging signals along $(\theta, \phi)$ will experience a frequency-diverse transfer function. We propose to exploit this property of the lens-loaded cavity and differentiate the DoA of an impinging signal based on the direction dependent gain. To test this, we measured the frequency-diverse transfer function and spatially quasi-orthogonal radiation fields of a lens-loaded cavity antenna within the frequency range of $27-29 \mathrm{GHz}$ (see 


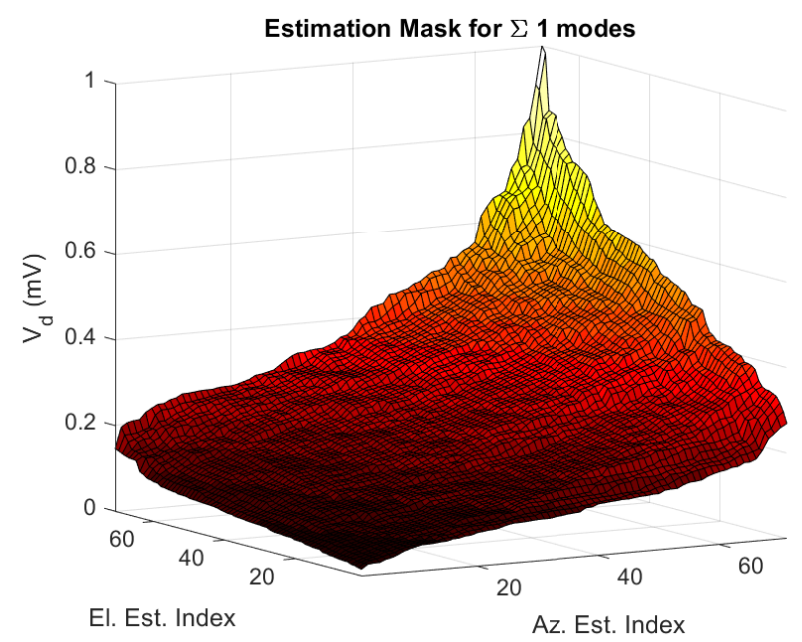

(a)

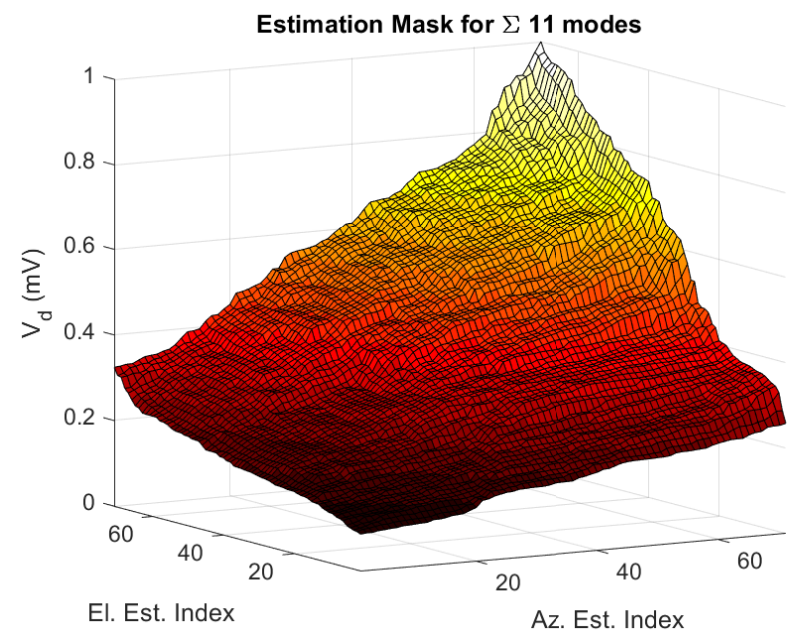

(b)

Fig. 4: Detector diode circuit output voltages indexed along azimuth and elevation FoV and sorted in ascending order to generate estimation masks when (a) 1 mode and (b) normalized sum of 11 modes are used (normalized color map scale).

Fig. 2(b)). The fields were recorded using a frequency sweep interval of $50 \mathrm{MHz}$, hence 41 radiation modes were captured within the $2 \mathrm{GHz}$ bandwidth. It should be noted that there are always measurement discrepancies and fabrication anomalies with complex structures like the one used in this investigation, however, the lens-loaded cavity inherently operates at quasirandomness of the radiation modes, and the fields recorded at the output of the cavity are very hardware specific. Hence, the measurement discrepancies are not considered to be a fundamental limit of the proposed approach. Another point to note here is that the fields at the output of the lens loaded cavity for a vertically polarized impinging signal are different from the fields observed for a horizontally polarized signal. Hence, measurements were performed separately for vertical and horizontal polarization. A vector sum of both polarization components results in defining the level of $P_{a v, d}$ at a load impedance of $50 \Omega$, measured using Keysight PNA 8361C. The voltage levels against $P_{a v, d}$ at the output of the estimator block

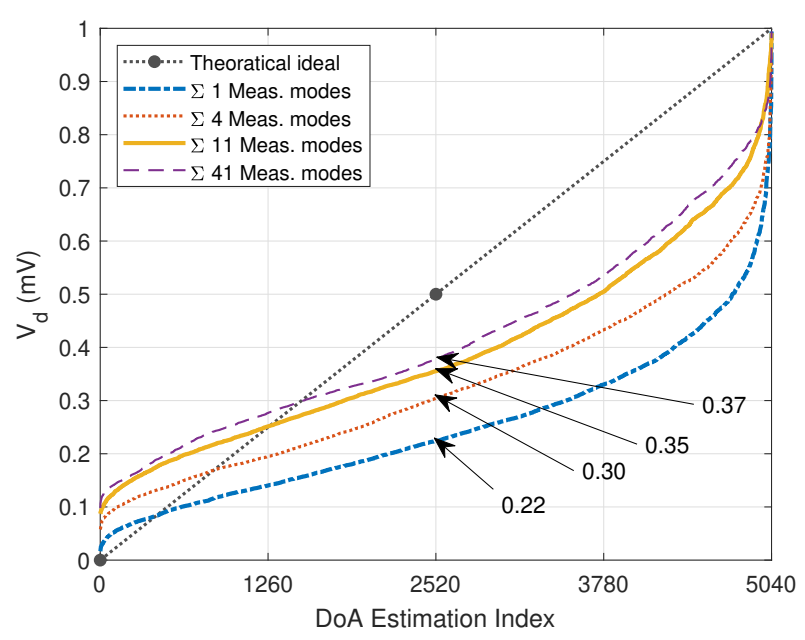

Fig. 5: Comparison between normalized sum of $V_{d}$ for multiple modes sorted in ascending order versus DoA estimation index.

in Fig. 1 are numerically evaluated in a post-processing step.

Output voltage maps (normalized to $1 \mathrm{mV}$ ) are presented in Fig. 3 against the impinging signal's measured $P_{a v, d}$. The discrimination between the voltage level for different combinations of azimuthal elevation FoV can be clearly seen. One important point to note is that for a given mode, e.g. mode 1 , the same normalized voltage level can be mistaken for more than one DoA which can lead to erroneous estimation. To overcome this, we propose the use of multiple modes to enhance the DoA estimation accuracy. For instance, a high number of distinct $V_{d}$ values are observed for each DoA when a normalized sum of more than $1 V_{d}$ mode masks are used compared to the normalized $V_{d}$ of 1 mode. For example, in Fig. 3 the normalized sum of $V_{d}$ for 4,11 and 41 modes is provided.

To further explain this, let us consider unique $V_{d}$ values indexed against DoA estimation and sorted in ascending order to generate a DoA estimation mask. If we use only 1 mode to generate an estimation mask, the possibility of more than 1 DoA estimation overlaps is higher compared to when 11 modes are used to create a similar estimation mask. This can be visualized in Fig. 4 where the estimation masks for both these cases are illustrated. If the same quantization interval is used to index the DoA for a given normalized $V_{d}$, then the DoA estimation using a greater number of modes will naturally be better. However, there is a limit to this enhancement. To demonstrate this, let us observe the total number of DoA estimation indexes based on the measurement results versus normalized $V_{d}$ in Fig. 4. In theory, half of the DoA estimation indexes should represent $V_{d}$ values from $0-0.5$, while the remaining half should represent $V_{d}$ from 0.5 - 1 . If we use 1 mode for DoA estimation, the majority of the DoA estimation indexes will be reserved for low $V_{d}$ values, and vice versa. This can be solved by using a greater number of modes. Recalling the previous example, if we use 11 modes, $50 \%$ of the DoA estimation indexes will represent $V_{d}$ values below 0.35 , compared to below 0.22 when only 1 mode is used. The higher the number of modes used, the lower the number 


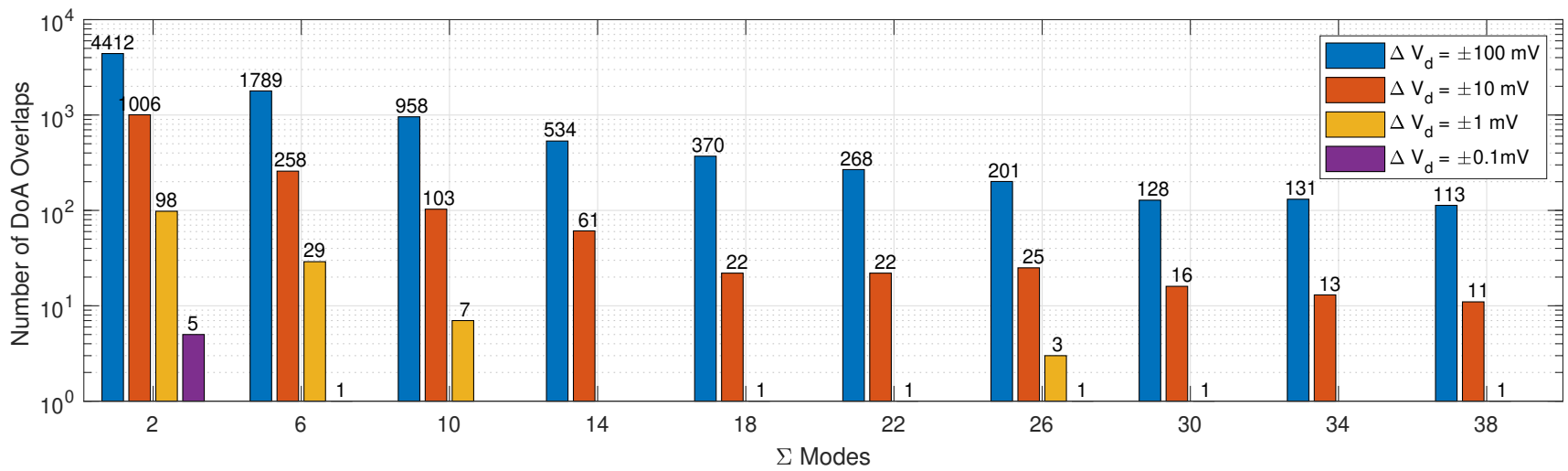

Fig. 6: Number of DoA estimation overlaps (errors) vs the number of modes used for DoA estimation, compared for multiple values of $\Delta V_{d}$.

of DoA estimation overlaps. Consulting Fig. 5, it is worth highlighting that although DoA estimation is better for the sum of 41 modes compared to the sum of 11 modes, the benefit is marginal. Based on this observation, there is a clear opportunity to conserve bandwidth, which is the most prominent feature of the proposed approach. The use of 11 modes requires only $0.55 \mathrm{GHz}$ of bandwidth as compared to $2.00 \mathrm{GHz}$ when 41 modes are used.

\section{BAndwidth-Sensitivity TRAde OfFS}

It has been established in the previous section that the proposed approach can provide benefit in terms of bandwidth conservation. This section analyzes the trade-off between bandwidth and estimator sensitivity. Fig. 6 presents the overall number of DoA estimation overlaps (i.e. errors) versus the normalized sum of modes used for the DoA estimation. Here, measured $P_{a v, d}$ values are used when the voltage resolution is varied, which depends upon the diode's $S_{\text {est }}$. For a low resolution $\Delta V_{d}$, e.g. at $\pm 100 \mathrm{mV}$, there are 4412 errors, which decreases to 534 when 14 modes are used. The number of errors are observed to go as low as 113 when 41 modes are used. The same trend can be observed for higher $\Delta V_{d}$ resolution. This trend highlights that by using a better resolution for $V_{d}$, where $V_{d}$ is directly proportional to the sensitivity, we can decrease the number of errors while operating at the same number of modes. For example, setting the number of modes to be 6 , as the $\Delta V_{d}$ is varied from $\pm 100 \mathrm{mV}$ to $\pm 0.1 \mathrm{mV}$, the number of errors decreases from 1789 to 1.

The choice of detector diode circuit is dependent upon the operational $P_{a v, c}$ and $P_{a v, d}$. Consider, for example, at $P_{a v, d}=$ $-30 \mathrm{dBm}$, the Anritsu 70KA50 detector provides $V_{d}=0.1 \mathrm{mV}$ with a load resistance is $1 \mathrm{k} \Omega$, hence the operational $S_{\text {est }}$ will be $100 \mathrm{mV} / \mathrm{mW}$. It is worth mentioning that detectors with low tangential signal sensitivity (TSS) and high $S_{\text {est }}$ should also be considered to achieve better DoA estimation accuracy within a given bandwidth (e.g. Pasternak PE8009-P with TSS $=-52 \mathrm{dBm}$ at $26 \mathrm{GHz}$ ). For detectors requiring higher input power, an additional RF amplification stage can be included before the matching network in Fig. 1, however, this should be implemented with caution as it may impact the overall linearity of the system. Note that the assumption that the spectrum of the incident plane-wave remains constant is valid as shown in
[16] since we are operating at a much higher frequency i.e. 28 $\mathrm{GHz}$, with respect to the the variation across center frequency i.e. less than $1 \mathrm{GHz}$.

\section{Limitations of The Proposed ApPROACH}

While the proposed approach marks a significant step towards achieving accurate DoA estimation using low complexity hardware, it does require some considerations. Firstly, the proposed approach requires the lens-loaded cavity to act only as a receiver, while an additional port will be required if the same cavity hardware is to be used as a transmitter. Secondly, calibration of the system in a controlled radio environment is required, especially when high-sensitivity and low bandwidth DoA estimation is to be achieved. A co-located antenna with uniform gain within the FoV is required for referencing $P_{a v, c}$ to accurately develop the DoA estimation mask at a given $S_{\text {est }}$. Thirdly, to achieve high sensitivity over smaller bandwidth, the estimator module requires precision radio hardware. This is especially true for the detector diode and the transition between the lens-loaded cavity and estimator module. Lastly, high linearity of detector diode circuit is essential to facilitate accurate DoA estimation within the dynamic range $P_{a v, d}$.

\section{Conclusions}

This letter has proposed a novel DoA estimation technique that utilizes a frequency-diverse lens-loaded cavity antenna and a mmWave receiver. The new approach makes use of a detector diode circuit with frequency-diverse antenna, to translate the DoA of an impinging signal into a detectable voltage level. The major contribution is the estimation technique that uses two distinct modules combined in a simplified fashion. A bandwidth conservation of $\sim 70 \%$ is achieved compared to computational DoA based estimation technique, at the cost of additional (sensitive) radio hardware. The trade-off between bandwidth and detector diode circuit sensitivity highlights the impact of sensitivity on the DoA estimation accuracy. As a result of its low complexity, the proposed system will find application in mmWave channel estimation, localization, and radio source positioning. 


\section{REFERENCES}

[1] F. Gao and A. B. Gershman, "A generalized ESPRIT approach to direction-of-arrival estimation," IEEE Signal Process. Lett., vol. 12, no. 3, pp. 254-257, 2005.

[2] X. Zhang, Y. Huang, C. Chen, J. Li, and D. Xu, "Reduced-complexity capon for direction of arrival estimation in a monostatic multiple-input multiple-output radar," IET Radar, Sonar Navig., vol. 6, no. 8, pp. 796801, 2012.

[3] H. Sim, S. Lee, S. Kang, and S.-C. Kim, "Enhanced DOA estimation using linearly predicted array expansion for automotive radar systems," IEEE Access., vol. 7, pp. 47 714-47 727, 2019.

[4] M. Mohanna, M. L. Rabeh, E. M. Zieur, and S. Hekala, "Optimization of music algorithm for angle of arrival estimation in wireless communications," NRIAG J. Astron. Geophys., vol. 2, no. 1, pp. 116-124, 2013.

[5] A. L. Kintz and I. J. Gupta, "A modified music algorithm for direction of arrival estimation in the presence of antenna array manifold mismatch," IEEE Trans. Antennas Propag., vol. 64, no. 11, pp. 4836-4847, 2016.

[6] E. Vlachos, J. Thompson, M. A. B. Abbasi, V. F. Fusco, and M. Matthaiou, "Robust estimator for lens-based hybrid MIMO with low-resolution sampling," in 2019 IEEE 20th Int. Workshop on Signal Process. Advances in Wireless Commun. (SPAWC). IEEE, 2019, pp. $1-5$.

[7] M. Giordani, M. Polese, A. Roy, D. Castor, and M. Zorzi, "A tutorial on beam management for 3GPP NR at mmWave frequencies," IEEE Commun. Surveys Tuts., vol. 21, no. 1, pp. 173-196, 2018.

[8] F. Shu, Y. Qin, T. Liu, L. Gui, Y. Zhang, J. Li, and Z. Han, "Lowcomplexity and high-resolution DOA estimation for hybrid analog and digital massive MIMO receive array," IEEE Trans. Commun., vol. 66, no. 6 , pp. $2487-2501,2018$.

[9] M. A. B. Abbasi, V. Fusco, and D. E. Zelenchuk, "Compressive sensing multiplicative antenna array," IEEE Trans. Antennas Propag., vol. 66, no. 11 , pp. $5918-5925,2018$

[10] M. Zhao, S. Zhu, J. Li, H. Shi, J. Chen, Y. He, and A. Zhang, "Frequency-diverse bunching metamaterial antenna for coincidence imaging," Mater, vol. 12, no. 11, p. 1817, 2019.

[11] O. Yurduseven, P. Flowers, S. Ye, D. L. Marks, J. N. Gollub, T. Fromenteze, B. J. Wiley, and D. R. Smith, "Computational microwave imaging using 3D printed conductive polymer frequency-diverse metasurface antennas," IET Microw. Antennas Propag., vol. 11, no. 14, pp. 1962 1969, 2017.

[12] T. Fromenteze, O. Yurduseven, M. F. Imani, J. Gollub, C. Decroze, D. Carsenat, and D. R. Smith, "Computational imaging using a modemixing cavity at microwave frequencies," Appl. Phys. Lett., vol. 106, no. 19, p. 194104, 2015.

[13] O. Yurduseven, M. A. B. Abbasi, T. Fromenteze, and V. Fusco, "Lensloaded coded aperture with increased information capacity for computational microwave imaging," Remote Sens., vol. 12, no. 9, p. 1531, 2020.

[14] O. Yurduseven, M. F. Imani, H. Odabasi, J. Gollub, G. Lipworth, A. Rose, and D. R. Smith, "Resolution of the frequency diverse metamaterial aperture imager," Prog. Electromagn. Res., vol. 150, pp. 97-107, 2015.

[15] O. Yurduseven, M. A. B. Abbasi, T. Fromenteze, and V. Fusco, "Frequency-diverse computational direction of arrival estimation technique," Sci. Rep., vol. 9, no. 1, pp. 1-12, 2019.

[16] M. Abbasi, V. Fusco, O. Yurduseven, and T. Fromenteze, "Frequencydiverse multimode millimetre-wave constant- $r$ lens-loaded cavity," Sci. Rep., vol. 10, no. 1, pp. 1-12, 2020.

[17] M. A. B. Abbasi, V. F. Fusco, H. Tataria, and M. Matthaiou, "Constant$\epsilon_{r}$ lens beamformer for low-complexity millimeter-wave hybrid MIMO," IEEE Trans. Microw. Theory Techn, vol. 67, no. 7, pp. 2894-2903, 2019. 